\title{
Coulomb effects on electromagnetic pair production in ultrarelativistic heavy-ion collisions
}

\author{
U. Eichmann, ${ }^{1}$ J. Reinhardt, ${ }^{1}$ S. Schramm, ${ }^{2}$ and W. Greiner ${ }^{1}$ \\ ${ }^{1}$ Institut für Theoretische Physik, Johann Wolfgang Goethe-Universität, D-60054 Frankfurt, Germany \\ ${ }^{2}$ Gesellschaft für Schwerionenforschung $\mathrm{mbH}$, D-64291 Darmstadt, Germany
}

(Received 6 July 1998)

\begin{abstract}
We calculate the asymptotic high-energy amplitude for electrons scattering at one ion, as well as at two colliding ions, by means of perturbation theory. We show that the interaction with one ion eikonalizes and that the interaction with two ions causally decouples. We are able to put previous results on perturbative grounds and propose further applications for the obtained rules for interactions on the light cone. We discuss the implications of the eikonal amplitude on the pair production probability in ultrarelativistic peripheral heavy-ion collisions. In this context the Weizsäcker-Williams method is shown to be exact in the ultrarelativistic limit, irrespective of the produced particles' mass. A new equivalent single-photon distribution is derived, which correctly accounts for Coulomb distortions. The impact on single-photon induced processes is discussed. [S1050-2947(99)00602-2]
\end{abstract}

PACS number(s): 11.80.-m, 25.75.-q, 12.20.-m

\section{INTRODUCTION}

At ultrarelativistic energies the theoretical treatment of scattering processes is extremely facilitated. On the one hand, the relevant equations themselves simplify, when terms of order $O\left(1 / \gamma^{2}\right)$ become negligible; on the other hand, the interactions simplify due to causality. In that way, high-energy scattering becomes analytically accessible.

Eikonal approximations or optical models usually are formulated for scattering of a highly energetic particle at a slow or static center $[1,2]$. We present a simple transformation of the covariant derivatives, that is used to easily solve the opposite case. In this way we can show that the $S$ matrix describing electron scattering at ultrarelativistic pointlike charges is determined by the gauge phase leading to the Dirac equation represented in the temporal gauge. We find that it naturally exhibits the same form as the well-known eikonal expression, as is expected by Lorentz invariance. Our results, therefore, coincide with previous calculations performed in this reference frame $[3,4]$.

The summation of ladder graphs is shown to eikonalize as well [5-7]. This was elegantly derived by kinematically decoupling the components of the scattering process, and Lorentz transforming into the respective rest frames [8], which inherently contains the advantages of a fast external potential.

Following a different approach we will exploit the same advantages. We follow a perturbative approach and directly approximate the external potential by its asymptotic highenergy limit, which amounts to saying that the longitudinal components of the exchanged photons can be discarded. In doing so, one can directly rederive the amplitude for the scattering at one center and even put the recent result of Segev and Wells [9] for the scattering amplitude for an electron moving in the field of two ultrarelativistic colliding ions on perturbative grounds. Moreover, one can go beyond the scope of their calculations to obtain additional insight.

The derivations in this paper are first formulated for electron scattering. Employing crossing invariance, they are extended to cover the physically more relevant process of electron-positron pair production. The search for exact ana- lytic expressions describing electron-positron pair production in heavy-ion collisions is motivated by the question whether Coulomb effects only play an inferior role at high energies. Such a conclusion might be drawn from a comparison between second-order perturbation theory results [10] and calculations employing Furry-Sommerfeld-Maue wave functions [11]. ${ }^{1}$

To easily assess the effects of Coulomb distortions, we state the problem in terms of the Weizsäcker-Williams method of equivalent quanta. We derive equivalent singlephoton distributions, which correctly account for Coulomb boundary conditions.

\section{SCATTERING OF AN ELECTRON OFF A FAST MOVING SOURCE}

\section{A. Transformation of the Dirac equation}

We are searching for the asymptotic scattering solution of a Dirac particle from a fast moving Coulomb potential in the limit of very large collision energy. In the Lorentz gauge the Liénard-Wiechert potentials for a point charge moving with uniform velocity $\beta$ in $+z$ direction read

$$
\begin{gathered}
A_{0}=-\frac{Z \alpha \gamma}{\sqrt{\gamma^{2}(z-\beta t)^{2}+\vec{x}_{\perp}^{2}}}, \\
A_{3}=\beta A_{0} .
\end{gathered}
$$

The equation of motion for the scattered particle becomes

$$
\left[\hat{\gamma}_{0}\left(i \partial_{t}-A_{0}\right)+\hat{\gamma}_{3}\left(i \partial_{z}+A_{3}\right)+\hat{\vec{\gamma}}_{\perp} \cdot i \vec{\nabla}_{\perp}-m\right] \psi=0 .
$$

We set $c=\hbar=1$. The charge $e$ of the electron was absorbed into the definition of the potential. We make use of the external field approximation, i.e., we assume that the source is not influenced by the scattered particle and moves on a straight line. This treatment will be justified if the mass of

\footnotetext{
${ }^{1}$ It should be mentioned, however, that the Coulomb distortions considered in these calculations only account for one ion, whereas the second ion enters as a perturbation.
} 
the source particle is very large. To simplify the Dirac equation (3) we use the operator identity [12]

$$
\left(i \partial_{x} \mp i \partial_{x} \ln \phi\right)^{n}=\phi^{ \pm 1}\left(i \partial_{x}\right)^{n} \phi^{\mp 1}
$$

to rewrite the covariant derivatives. We must introduce two fields $\phi^{\prime}$ and $\phi$ for the space and the time component of the vector potential $A^{\mu}$,

$$
\begin{gathered}
A_{0}=i \partial_{t} \ln \phi, \\
A_{3}=i \partial_{z} \ln \phi^{\prime} .
\end{gathered}
$$

The auxiliary fields are determined to be

$$
\phi(x)=\exp \left(-i \int_{-\infty}^{t} d t^{\prime} A_{0}(x)\right)
$$

and

$$
\phi^{\prime}=\phi^{-\beta^{2}}
$$

Inserting Eq. (5) into Eq. (3) we arrive at the transformed Dirac equation

$$
\begin{aligned}
& {\left[\hat{\gamma}_{0} i \partial_{t}+\hat{\gamma}_{3}\left(i \partial_{z}-\frac{1}{\beta^{2} \gamma^{2}} A_{3}\right)\right.} \\
& \left.+\hat{\vec{\gamma}}_{\perp} \cdot\left(i \vec{\nabla}_{\perp}+i \operatorname{grad}_{\perp} \ln \phi\right)-m\right] \widetilde{\psi}=0,
\end{aligned}
$$

where

$$
\widetilde{\psi}=\psi / \phi=\exp \left(i \int_{-\infty}^{t} d t^{\prime} A_{0}\right) \psi
$$

The operator identity has led to the elimination of the scalar part of the vector potential, i.e., to the temporal gauge, $A_{0}^{\prime}$ $=0$. This will be of particular importance in Sec. VI. In the ultrarelativistic limit terms of the order $O(1 / \gamma)$ are neglected and we end up with a Dirac equation coupled to a purely transverse vector potential $\vec{A}^{\prime}{ }_{\perp}=i \operatorname{grad}_{\perp} \ln \phi$ $=\int_{-\infty}^{t} d t^{\prime} \operatorname{grad}_{\perp} A_{0}$, which is the negative time integral of the transverse electric field. From classical electrodynamics one knows that the time integral of the transverse electric field is given by

$$
\int_{-\infty}^{\infty} d t \vec{E}_{\perp}=-2 Z \alpha \frac{\vec{x}_{\perp}}{\beta x_{\perp}^{2}} .
$$

This implies that

$$
\int_{-\infty}^{\infty} d t A_{0}=\frac{1}{\beta} Z \alpha \ln \vec{x}_{\perp}^{2}+\mathcal{C}
$$

$\mathcal{C}$ is an infinite quantity, which expresses the divergence of the phases in Coulomb scattering. Using Eqs. (6) and (1) it is easy to show that the transverse vector potential $\vec{A}_{\perp}^{\prime}$ exhibits a Heaviside step function dependence $\sim \theta(t-z) \vec{x}_{\perp} / x_{\perp}^{2}$ in the limit of very large $\gamma$. Since $t$ and $\gamma$ enter symmetrically in the integral, the limit $\gamma \rightarrow \infty$ corresponds to sending the upper bound of the integral in Eq. (6) to infinity. Therefore, all of the above applies, giving

$$
\lim _{\gamma \rightarrow \infty} A_{0}=Z \alpha \delta(z-t) \ln \vec{x}_{\perp}^{2}+\mathcal{C}^{\prime} .
$$

The Coulomb phase $\mathcal{C}^{\prime}$ will, in general, depend on $z$ and $t$. It can be removed by an additional gauge transformation as is easily seen:

$$
\widetilde{\psi}^{\prime}=\exp \left(-i \int_{-\infty}^{t} d t^{\prime} \mathcal{C}^{\prime}\right) \widetilde{\psi}=e^{+i Z \alpha \theta(t-z) \ln \vec{x}_{\perp}^{2}} \psi .
$$

The gauge transformation (11) was first applied by Aichelburg and Sexl [13]. The removal of the Coulomb phase yields a short-range potential allowing for asymptotic planewave solutions (see Appendix A).

For $t \neq z$ the $t$ and $z$ dependence in both the transverse vector potential and the transformed spinor $\widetilde{\psi}$ vanish in the limit $\gamma \rightarrow \infty$. By inverse transformation we find that $\psi$ solves a free Dirac equation on either side of the light front $t=z$ and can only differ by a phase.

The transformed wave function $\tilde{\psi}$ has the advantage of being continuous across the surface defined by $t=z$. In contrast, the wave function $\psi$ exhibits a discontinuous behavior at the light front. There is a jump in that component of $\psi$ that couples to $\hat{\gamma}_{-}=\hat{\gamma}_{0}-\hat{\gamma}_{3}$, the matrix structure of the interaction in the limit $\gamma \rightarrow \infty$. Using this property one directly finds for $\hat{\gamma}_{-} \psi$ at the discontinuity,

$$
\hat{\gamma}_{-} \psi\left(t-z=0^{+}\right)=e^{-i Z \alpha \ln \dot{x}_{\perp}^{2}} \hat{\gamma}_{-} \psi\left(t-z=0^{-}\right),
$$

where we ignored the irrelevant constant phase $\mathcal{C}$. ${ }^{2}$ The complement $\hat{\gamma}_{+} \psi$ of these spinor components, where $\hat{\gamma}_{+}$ $=2 \hat{\gamma}_{0}-\hat{\gamma}_{-}=\hat{\gamma}_{0}+\hat{\gamma}_{3}$, is continuous at $t=z$. On either side of the discontinuity both parts of the spinor are coupled via the free Dirac equation.

By application of the Lehmann-Symanzik-Zimmermann reduction formula one finds, in general, that at very large scattering energies the $S$ operator is determined by the function $\phi$, in which we recognize the well-known eikonal expression. We note that because of the identity (4) this result holds independently of the power of the momentum entering in the respective wave equation. For that reason the expressions for the $S$ matrices for, e.g., spinor or scalar particles only differ by an overall factor [14]. We see that the obtained $S$ operator is a unitary operator since it is connected to the gauge phase $\phi$. It agrees with the first term of the Magnus expansion of the time-evolution operator [15], since in the ultrarelativistic limit the considered gauge-transformed interaction is compressed to an infinitely short time span. In fact

\footnotetext{
${ }^{2}$ The effect of the potential (10) also can be described within the Aichelburg-Sexl metric $[4,13]$. Two field-free regions of space-time meet at $z=t$, such that (the superscripts $>$ and $<$ denote $t>z$ and $t<z, \quad$ respectively), $\quad \vec{x}_{\perp}^{>}=\vec{x}_{\perp}^{<}, z^{>}=z^{<}-Z \alpha / p_{-} \ln x_{\perp}^{2}, t^{>}=t^{<}$ $-Z \alpha / p_{-} \ln x_{\perp}^{2}$. The result (12) is then easily obtained by simply substituting the above expressions into the plane wave at $t>z$.
} 
the first-order Magnus expansion, i.e., the eikonal approximation, sufficiently describes the scattering process as long as the duration of the electromagnetic interaction $t_{i n t}$ $\sim x_{\perp} / \gamma$ is short compared to the typical transition time for the scattering process $t_{\text {trans }} \sim 1 /\left|E_{f}-E_{i}\right| \sim 1 / \omega$. The condition can be cast in the form $x_{\perp} \ll \gamma / \omega$. It is trivially fulfilled at infinite scattering energies, and the eikonal approximation becomes exact. At finite $\gamma$, we have to consider an adequate screening of large transverse separations. We will elaborate this condition and the cutoff in Sec. VII and in Appendix A.

Let us explicitly write down an expression for the $S$ matrix, which follows from the above reasoning. The left-hand side of Eq. (12) can be expanded in plane waves. Since we consider scattering at the negative light front, we must substitute $d^{3} x \rightarrow d x_{+} d^{2} x_{\perp} \quad$ [16] and accordingly $d^{3} p$ $\rightarrow d p_{-} d^{2} p_{\perp}$. The expansion coefficient gives the $S$ matrix for electron scattering in momentum space ( $p$ and $p^{\prime}$ denote the initial and final momentum)

$$
\begin{aligned}
S\left(p^{\prime}, p\right)= & 2 \pi \delta\left(p_{-}^{\prime}-p_{-}\right)\left[F_{p^{\prime} p}\left(e^{-i Z \alpha \ln \vec{x}_{\perp}^{2}}-1\right)\right. \\
& \left.+(2 \pi)^{2} \delta^{2}\left(\vec{p}^{\prime}{ }_{\perp}-\vec{p}_{\perp}\right)\right] \frac{m}{\sqrt{p_{0}^{\prime} p_{0}}} \bar{u}\left(p^{\prime}\right) \hat{\gamma}_{-} u(p) .
\end{aligned}
$$

$u$ denotes the electron unit spinor, which is normalized according to $\bar{u}(p) u(p)=1 . \quad F_{p^{\prime} p}$ abbreviates the Fourier transform with respect to the transverse coordinates, taken at the momentum $\left(\vec{p}_{\perp}^{\prime}-\vec{p}_{\perp}\right)$

$$
F_{p^{\prime} p}\left(f\left(\vec{x}_{\perp}\right)\right)=\int d^{2} x_{\perp} e^{-i \vec{x}_{\perp} \cdot\left(\vec{p}_{\perp}^{\prime}-\vec{p}_{\perp}\right)} f\left(\vec{x}_{\perp}\right) .
$$

We note that the negative light-cone momentum $p_{-}=p_{0}$ $-p_{3}$ is conserved in the scattering. The positive light cone momentum $p_{+}=p_{0}+p_{3}$ is fixed by the mass shell condition $p_{+} p_{-}=m^{2}+\vec{p}_{\perp}^{2}$. Equation (13) represents a well-known result that was previously derived in, e.g., $[1,4,3,9]$. The first term in the square brackets in Eq. (13) corresponds to the $T$ matrix. The exact amplitude for electron scattering at an ultrarelativistic pointlike charge, moving in $+z$ direction, therefore, reads ${ }^{3}$

$$
\begin{aligned}
& A_{p^{\prime} p}=2 \pi \delta\left(p_{-}^{\prime}-p_{-}\right) F_{p^{\prime} p}\left(e^{-i Z \alpha \ln \vec{x}_{\perp}^{2}}-1\right) \bar{u}\left(p^{\prime}\right) \hat{\gamma}_{-} u(p) \\
& =-i 8 \pi^{2} Z \alpha \delta\left(p_{-}^{\prime}-p_{-}\right) \frac{1}{t} \frac{\Gamma(1-i Z \alpha)}{\Gamma(1+i Z \alpha)} \\
& \times e^{i Z \alpha \ln (-t / 4)} \bar{u}\left(p^{\prime}\right) \hat{\gamma}_{-} u(p) \text {. }
\end{aligned}
$$

${ }^{3}$ Note the striking similarity between Eq. (15) and the nonrelativistic (Schrödinger) amplitude for Coulomb scattering [17],

$$
f(\theta)=-\frac{1}{2 k^{2} \sin ^{2} \frac{\theta}{2}} \frac{\Gamma\left(1-\frac{i}{k}\right)}{\Gamma\left(1+\frac{i}{k}\right)} e^{(i / k) \ln \sin ^{2} \theta / 2} .
$$

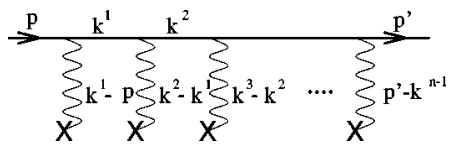

FIG. 1. Scattering of an electron at an external potential.

Here we used the invariant squared momentum transfer $t$ $=\left(p^{\prime}-p\right)^{2}$, which approaches $t \approx-\left(\vec{p}_{\perp}^{\prime}-\vec{p}_{\perp}\right)^{2}$ in the limit $\gamma \rightarrow \infty$.

\section{B. Perturbative approach}

In this section we want to derive the eikonal form of the $T$ matrix via perturbation theory. Several approximations are necessary to obtain the eikonal form, namely, the neglect of the longitudinal components of the photon momentum, the conservation of the photon light cone momentum, as well as the simplification of the matrix structure of the interaction [5]. The calculation shows that these approximations are the counterparts of the requirement of a negligible longitudinal vector potential and the step function dependence of the transverse vector potential. Having this in mind we directly use the asymptotic high-energy expression of the potential. We then evaluate the terms of the perturbation series for the external-field scattering problem depicted by the Feynman graphs of Fig. 1.

The potential entering into our calculations is of the form

$$
A_{0}(x)=A_{3}(x)=\delta(z-t) V_{\perp}\left(\vec{x}_{\perp}\right)
$$

In the following calculations it will not be necessary to specify the explicit form of $V_{\perp}\left(\vec{x}_{\perp}\right)$. Problems arising from the logarithmic potential obtained in the last section will be discussed in Sec. IV. The Feynman propagator describing the internal electron lines reads in terms of light-cone and transverse momenta

$$
\begin{aligned}
S_{F}(p) & =\frac{1}{\hat{\gamma}_{0} p_{0}-\hat{\vec{\gamma}} \cdot \vec{p}-m+i \epsilon} \\
& =\frac{\frac{1}{2}\left(\hat{\gamma}_{-} p_{+}+\hat{\gamma}_{+} p_{-}\right)-\hat{\vec{\gamma}}_{\perp} \cdot \vec{p}_{\perp}+m}{p_{+} p_{-}-p_{\perp}^{2}-m^{2}+i \epsilon} .
\end{aligned}
$$

The amplitude for electron scattering in first-order perturbation theory is

$$
A_{p^{\prime} p}^{(1)}=(2 \pi)(-i) \delta\left(p_{-}^{\prime}-p_{-}\right) F_{p^{\prime} p}\left(V_{\perp}\left(\vec{x}_{\perp}\right)\right) \bar{u}\left(p^{\prime}\right) \hat{\gamma}_{-} u(p)
$$

In second-order perturbation theory the amplitude reads 


$$
\begin{aligned}
A_{p^{\prime} p}^{(2)}= & \int \frac{d k_{+} d k_{-} d^{2} k_{\perp}}{(2 \pi)^{4}}(2 \pi)^{2}(-i)^{2} i \delta\left(k_{-}-p_{-}\right) \\
& \times \delta\left(p_{-}^{\prime}-k_{-}\right) \frac{k_{-}}{k_{-} k_{+}-k_{\perp}^{2}-m^{2}+i \epsilon} F_{k p}\left[V_{\perp}\left(\vec{x}_{\perp}\right)\right] \\
& \times F_{p^{\prime} k}\left[V_{\perp}\left(\vec{x}_{\perp}\right)\right] \bar{u}\left(p^{\prime}\right) \hat{\gamma}_{-} u(p) \\
= & (2 \pi)(-i)^{2} \delta\left(p_{-}^{\prime}-p_{-}\right) \\
& \times \frac{1}{2} F_{p^{\prime} p}\left[V_{\perp}^{2}\left(\vec{x}_{\perp}\right)\right] \bar{u}\left(p^{\prime}\right) \hat{\gamma}_{-} u(p)
\end{aligned}
$$

The $k_{+}$integral in Eq. (19) drops out using the symbolic substitution

$$
1 /(k+i \epsilon) \rightarrow P(1 / k)-i \pi \delta(k)
$$

since the principal value integral $P$ vanishes. It is interesting to note that the simple structure of the results (18) and (19) is retained if one goes to higher orders of perturbation theory. The $n$ th-order amplitude factorizes into $n-1$ integrals of the form (19), which leads to

$$
\begin{aligned}
A_{p^{\prime} p}^{(n)}= & (2 \pi)(-i)^{n} \delta\left(p_{-}^{\prime}-p_{-}\right) \frac{1}{n !} F_{p^{\prime} p}\left[V_{\perp}^{n}\left(\vec{x}_{\perp}\right)\right] \\
& \times \bar{u}\left(p^{\prime}\right) \hat{\gamma}_{-} u(p) .
\end{aligned}
$$

This result is obtained by symmetrizing the $n-1$ integrals over the positive light cone momenta yielding the expression $(-i 2 \pi)^{n-1} / n ! \Pi_{i} \delta\left(k_{+}^{i}\right)$ [5]. This corresponds to reconsidering the different time orderings and finally dividing by $n$ ! to prevent double counting. This symmetrization procedure directly shows that the principal value terms in Eq. (20) do not contribute.

Using Eq. (21) the perturbation series can be summed up to yield the result

$$
A_{p^{\prime} p}=2 \pi \delta\left(p_{-}^{\prime}-p_{-}\right) \mathcal{T}\left(\vec{p}_{\perp}^{\prime}-\vec{p}_{\perp}\right) \bar{u}\left(p^{\prime}\right) \hat{\gamma}_{-} u(p)
$$

Here we defined the momentum transfer function

$$
\mathcal{T}\left(\vec{p}_{\perp}^{\prime}-\vec{p}_{\perp}\right)=F_{p^{\prime} p}\left(e^{-i V_{\perp}\left(\vec{x}_{\perp}\right)}-1\right)
$$

with

$$
V_{\perp}\left(\vec{x}_{\perp}\right)=\int_{-\infty}^{+\infty} d t A_{0}(x)
$$

this result reproduces the eikonal form.

For $V_{\perp}\left(\vec{x}_{\perp}\right)=Z \alpha \ln x_{\perp}^{2}, \mathcal{T}\left(\vec{p}^{\prime}{ }_{\perp}-\vec{p}_{\perp}\right)$ reads [see Eq. (15) and Appendix D]

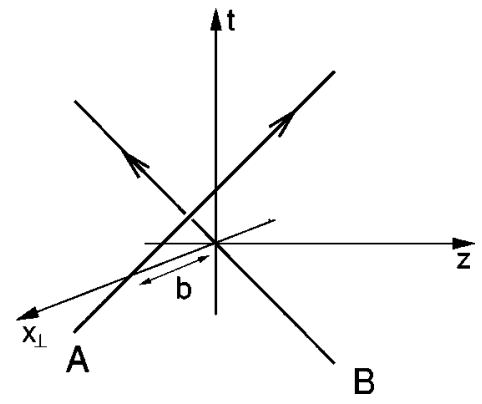

FIG. 2. Trajectories of two lightlike ions colliding with an impact parameter $\vec{b}$. The $x_{\perp}$ axis symbolically denotes the transverse plane. The $\vec{b}$ dependence of Eq. (27) is absorbed in the definitions of $V_{\perp}^{A, B}\left(\vec{x}_{\perp}\right)$.

$$
\mathcal{T}\left(\vec{p}_{\perp}^{\prime}-\vec{p}_{\perp}\right)=\pi \frac{\Gamma(1-i \alpha Z)}{\Gamma(i \alpha Z)}\left(\frac{4}{\left(\vec{p}_{\perp}^{\prime}-\vec{p}_{\perp}\right)^{2}}\right)^{1-i \alpha Z}
$$

The cross section for this scattering process is found to be exactly the Mott formula for Coulomb scattering of ultrarelativistic electrons at a static source, Lorentz transformed into the electron's rest frame. Such kind of agreement between the exact result and the first-order perturbation theory is also found in the nonrelativistic case, and is known as one of the peculiarities of the Coulomb field.

The well-established eikonalization of the scattering amplitude and thus the reduction to Mott's result imply that in the high-energy limit the electron and the positron Coulomb scattering cross section become identical. This behavior of the cross section at ultrarelativistic energies confirms the Pomeranchuk theorem [18].

The scattering process can be described in terms of the single exchange of an "effective photon" according to the modified potential (see also [19])

$$
A_{0}(x)=A_{3}(x)=\delta(z-t)\left[\left(\frac{1}{x_{\perp}}\right)^{2 i Z \alpha}-1\right] \text {. }
$$

\section{SOLUTION IN THE FIELD OF TWO COLLIDING IONS}

In the c.m. frame, the potential of two ultrarelativistic colliding ions $A$ and $B$ moving in $+z$ and $-z$ direction, cf. Fig. 2, reads

$$
A_{0 / 3}(x)=\delta(z-t) V_{\perp}^{A}\left(\vec{x}_{\perp}\right) \pm \delta(z+t) V_{\perp}^{B}\left(\vec{x}_{\perp}\right) .
$$

The identity (4) can also be applied to potentials given by a superposition as is easily verified,

$$
\left(i \partial_{x} \mp i \sum \partial_{x} \ln \phi_{i}\right)^{n}=\left(\prod \phi_{i}\right)^{ \pm 1}\left(i \partial_{x}\right)^{n}\left(\prod \phi_{i}\right)^{\mp 1}
$$




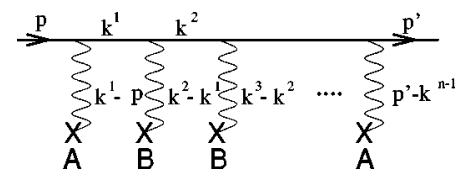

FIG. 3. Same as Fig. 1 but considering two ions $A$ and $B$ as external sources. This diagram does not contribute in the highenergy limit.

Since in the case of Eq. (27) we have two discontinuities, the asymptotic solution is not obtained as easily as in Sec. II A. It has been obtained recently by Segev and Wells [9]. A slightly modified derivation is shown in Appendix B.

It is found that the two ions couple to distinct components of the electron spinor. We show in this section how this behavior follows from perturbation theory and how it is interpreted consistently.

We have to consider several new diagrams describing the alternate interaction of the electron with both ions. We find that the contribution to the amplitude from an arbitrary number of interactions with one ion, which are sandwiched between interactions with the other ion (see Fig. 3), vanishes. The reason is that we end up with an integral of the form

$$
A \sim \int \frac{d k_{ \pm}}{\left(k_{ \pm} p_{\mp}-\vec{k}_{\perp}^{2}-m^{2}+i \epsilon\right)\left(k_{ \pm} p_{\mp}^{\prime}-\vec{k}_{\perp}^{\prime 2}-m^{2}+i \epsilon\right)}=0,
$$

which vanishes since the contour can be closed in the upper half plane, where the integrand is analytic. The integral runs over the positive (negative) light cone momentum, if the sandwiched photons mediate between the electron and the ion moving in the $-z(+z)$ direction. The two factors in the denominator stem from those electron propagators that are adjacent to the outer, enveloping, photons. $\vec{k}_{\perp}$ and $\overrightarrow{k^{\prime}} \perp$ correspond to the transverse photon momenta that are transferred to these electron lines.

In the ultrarelativistic limit the electron will, therefore, interact with the ions separately (see Fig. 4). The separate interactions of the electron with the two ions $A$ and $B$ are linked in the following way:

$$
\begin{aligned}
A_{p^{\prime} p}^{\text {tot }}= & \int \frac{d^{2} k_{\perp}}{(2 \pi)^{2}} \mathcal{T}_{B}\left(\vec{p}^{\prime}{ }_{\perp}-\vec{k}_{\perp}\right) \mathcal{T}_{A}\left(-\vec{p}_{\perp}+\vec{k}_{\perp}\right) e^{i\left(-\vec{p}_{\perp}+\vec{k}_{\perp}\right) \cdot \vec{b}} \\
& \times\left(\bar{u}\left(p^{\prime}\right) \frac{-\hat{\vec{\alpha}}_{\perp} \cdot \vec{k}_{\perp}+\gamma_{0} m}{p_{+}^{\prime} p_{-}-k_{\perp}^{2}-m^{2}+i \epsilon} \hat{\gamma}_{+} u(p)\right. \\
& \left.+\bar{u}\left(p^{\prime}\right) \frac{-\hat{\vec{\alpha}}_{\perp} \cdot\left(\vec{p}_{\perp}+\vec{p}_{\perp}^{\prime}-\vec{k}_{\perp}\right)+\gamma_{0} m}{p_{-}^{\prime} p_{+}-\left(\vec{p}_{\perp}+\vec{p}_{\perp}^{\prime}-k_{\perp}\right)^{2}-m^{2}+i \epsilon} \hat{\gamma}_{-} u(p)\right) .
\end{aligned}
$$

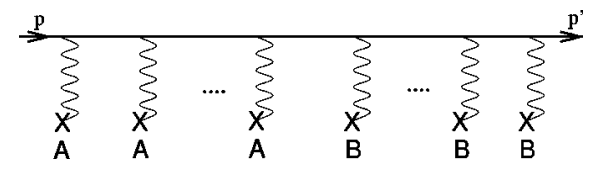

FIG. 4. Class of Feynman graphs contributing in the highenergy limit.
Here we have already added both possible time orderings. $\mathcal{T}_{A}$ and $\mathcal{T}_{B}$ are the momentum transfer functions defined in Eq. (23) for the interactions with ion $A$ and $B$, respectively. The single-ion scattering amplitudes have already been subtracted since they do not contribute to pair production which is finally envisaged. According to the shift theorem for Fourier transforms, the whole impact parameter dependence of the momentum transfer function $\mathcal{T}_{A}$ (see Fig. 2) is expressed by the factor $e^{i\left(-\vec{p}_{\perp}+\vec{k}_{\perp}\right) \cdot \vec{b}}$. Expression (30) can also be derived by using the discontinuous behavior of the wave function at the light fronts (see Appendix B) and corresponds to the result of Segev and Wells [9].

To understand the decoupling property, one has to consider the Dirac matrix structure of the potential. To this end we write the Dirac equation in the following form:

$$
\left[i \partial_{t}+\hat{\vec{\alpha}} \cdot i \vec{\nabla}-\hat{\gamma}_{0} m-\left(1 \pm \beta \hat{\alpha}_{z}\right) A_{0}\right] \psi=0
$$

where the sign depends upon the direction of motion and $A_{0}$ is given by Eq. (1). In the limit $\beta \rightarrow 1$ the operators (1 $\left.\pm \beta \hat{\alpha}_{z}\right) / 2$ become orthogonal projection operators [9]. The action of these operators becomes clear if one recalls the standard form of Lorentz transformations [18] in spinor space,

$$
\psi^{\prime}\left(x^{\prime}\right)=e^{-(i / 4) \sigma_{\alpha \beta} y^{\alpha \beta}} \psi(x),
$$

where $\sigma_{\alpha \beta}=(i / 2)\left[\hat{\gamma}_{\alpha}, \hat{\gamma}_{\beta}\right]$. The exponent represents the product of the rapidity vector $\vec{y}$ times the generators of the Lorentz transformation. For a boost in the $+z$ direction Eq. (32) simplifies to

$$
\psi^{\prime}\left(x^{\prime}\right)=e^{-(y / 2) \hat{\alpha}_{z}} \psi(x)=\cosh \left(\frac{y}{2}\right)\left[1-\tanh \left(\frac{y}{2}\right) \hat{\alpha}_{z}\right] \psi(x)
$$

A Lorentz-transformed vector acting in spinor space can therefore directly be obtained by the Lorentz transformation (32) accounting for the vectorial nature of the transformed object with a factor 2 in the exponent. See, for example, the action of the retarded Coulomb potential (1) on Dirac spinors, Eq. (31).

The operators $\left(1 \pm \hat{\alpha}_{z}\right)$ are thus $1 / \gamma$ times a Lorentz transformation with effectively infinite rapidity. These operators project the Dirac spinors onto causally disconnected subspaces of the Hilbert space. Therefore it is causally impossible for the Dirac spinor to communicate alternately with both ions. Since the matrix structure of the true interaction is given by $\left(1 \pm \beta \hat{\alpha}_{3}\right) \approx\left(1 \pm \hat{\alpha}_{3}\right) \mp \hat{\alpha}_{3} / 2 \gamma^{2}$, the leading corrections to this behavior are suppressed with $1 / \gamma^{2}$.

Due to this causal decoupling property, the exact expressions for the interaction of an electron with two colliding ultrarelativistic ions maintains the structure of the twophoton graph, containing, however, the exchange of effective photons according to Eq. (26). 


\section{MATHEMATICAL DISCUSSION}

In the previous sections the potential of a fast moving charge has been substituted by its asymptotic high-energy expression. From a mathematical (and also a physical) point of view this is a problematic limit, since the required transformation is not an element of the Lorentz group. Furthermore, the potential (1), a bounded operator in Hilbert space, gets transformed into an unbounded operator, and finally the number of spatial dimensions gets reduced from three to two.

The ansatz directly reflects the approximations made by Chang and $\mathrm{Ma}$ [5] who neglected the longitudinal components of the photon momentum, giving the $\delta$ functions for the respective conserved light cone momenta. The abovementioned problems emerge here in the fact that the longitudinal components of the photon momentum never really vanish.

All approximations allow the well-known conclusion that the eikonal expression can be regarded as the contribution of all ladder diagrams in the high-energy limit and that it is completely compatible with a perturbative calculation. In view of this result it should not be surprising that it is possible (in principle) to regain the first-order perturbation theory from the eikonal expression.

Note, however, that the perturbative derivation did not require us to specify either a transverse part of the interaction, or its explicit Fourier transform. The first point may serve to generalize the validity of the result to any function $V_{\perp}\left(\vec{x}_{\perp}\right)$. The Fourier transform in two dimensions of the considered logarithmic potential is, however, a nontrivial procedure. For that reason the deduction of the smallcoupling limit $(Z \alpha \rightarrow 0)$ of Eq. (15) must be treated with special care.

To calculate the small-coupling limit, one may recall that although two-dimensional massless fields are ill-defined objects, the exponential of these fields is not. Naive Taylor expansion of the Fourier transformed eikonal amplitude does, however, not yield the Fourier transform of the logarithm as the linear term. This peculiarity is due to the fact that the linearity of the Fourier transform is strictly guaranteed only for the action on finite sums. In the case of an infinite series like the Taylor expansion of the exponential function, Taylor expansion and Fourier transformation do not commute. Although it is therefore not justified to identify the different terms of the Taylor expansion with the Fourier transforms of the powers of the logarithm, the first term corresponds to the high-energy limit of the Fourier transform of the retarded Coulomb potential, but this is rather accidental.

Nevertheless, the correct Fourier transform of the logarithm in two dimensions can be obtained by Taylor expansion of the eikonal amplitude, but the limit $Z \alpha \rightarrow 0$ has to be taken after having integrated the expression with a test function [20]

$$
\begin{aligned}
& \int d^{2} x_{\perp} e^{-i \vec{k}_{\perp} \cdot \vec{x}_{\perp}} \ln \vec{x}_{\perp}^{2} \\
& =\lim _{i Z \alpha \rightarrow 0} \frac{d}{d(i Z \alpha)}\left[\pi \frac{\Gamma(1-i \alpha Z)}{\Gamma(i \alpha Z)}\left(\frac{4}{\vec{k}_{\perp}^{2}}\right)^{1-i \alpha Z}\right] .
\end{aligned}
$$

The error that is made in the naive calculation corresponds to the neglection of the Coulomb boundary conditions. This is seen as follows: We rewrite the logarithm as the time integral over the gauge-transformed Coulomb potential (see Appendix D). Recall that the gauge transformation removes an infinite quantity, which is related to the divergent Coulomb phase. The Fourier transform is then found to be $[21,22]$

$$
\begin{aligned}
& \int d^{2} x_{\perp} e^{-i \vec{k}_{\perp} \cdot \vec{x}_{\perp} \ln \vec{x}_{\perp}^{2}} \\
& =\lim _{\lambda \rightarrow 0} 4 \pi\left[\frac{-1}{\vec{k}_{\perp}^{2}+\lambda^{2}}+\pi \delta^{2}\left(\vec{k}_{\perp}\right) \ln \left(\frac{\mu^{2}}{\lambda^{2}}\right)\right],
\end{aligned}
$$

with $\lambda=\omega / \gamma, \mu=2 / e^{C}$. The correct treatment of this result again requires the limit to be taken after integrating the expression with a test function. The second term on the righthand side arises from the gauge transformation applied to the potential and thus accounts for the Coulomb distortions. Discarding the second term and taking the limit directly again leads to the discussed fortuitous agreement with the Fourier transform of the retarded Coulomb potential.

In view of these results, one may conclude that the correct small-coupling limit of the scattering amplitude in momentum space cannot be found by a naive Taylor expansion of the Fourier transformed $T$ matrix and does not agree with first-order perturbation theory. According to Eq. (35) this is simply based on the fact that the gauge transformed potential correctly accounts for Coulomb boundary conditions.

In the following we want to investigate how the correct treatment of Coulomb boundary conditions in all orders of perturbation theory influences the cross section of the scattering process.

\section{IMPLICATIONS ON THE PAIR-PRODUCTION CROSS SECTION}

In the field of two ultrarelativistic colliding pointlike nuclei, the exact scattering amplitude was shown to retain the structure of the second-order perturbative result, due to the causal decoupling property. Each interaction can be described by the modified potential (26). Accounting for both time orderings, the amplitude is given by Eq. (30).

We now use the crossing invariance of the amplitude to apply the obtained result to electron-positron pair production. The initial electron four momentum $p$ has then to be replaced by the negative final positron momentum $p \rightarrow$ $-p^{p}$. The final electron momentum will be denoted by $p^{\prime}$ $=p^{e}$. With Eq. (30) we obtain for the pair-production probability 


$$
\begin{aligned}
& \frac{d \sigma}{d^{2} b}=\left|A_{p^{\prime} p}^{t o t}\right|^{2} \frac{m d^{3} p^{e}}{(2 \pi)^{3} E^{e}} \frac{m d^{3} p^{p}}{(2 \pi)^{3} E^{p}} \\
& =\frac{m d^{3} p^{e}}{(2 \pi)^{3} E^{e}} \frac{m d^{3} p^{p}}{(2 \pi)^{3} E^{p}} \int \frac{d^{2} k_{\perp}}{(2 \pi)^{2}} \int \frac{d^{2} k_{\perp}^{\prime}}{(2 \pi)^{2}} \mathcal{T}_{B}\left(\vec{p}_{\perp}^{e}-\vec{k}_{\perp}\right) \mathcal{T}_{B}^{*}\left(\vec{p}_{\perp}^{e}-\vec{k}_{\perp}^{\prime}\right) \mathcal{T}_{A}\left(\vec{k}_{\perp}+\vec{p}_{\perp}^{p}\right) \mathcal{T}_{A}^{*}\left(\vec{k}_{\perp}^{\prime}+\vec{p}_{\perp}^{p}\right) e^{i\left(\vec{k}_{\perp}^{\prime}-\vec{k}_{\perp}\right) \cdot \vec{b}} \\
& \times\left(\bar{u}\left(p^{e}\right) \frac{-\hat{\vec{\alpha}}_{\perp} \cdot \vec{k}_{\perp}+\gamma_{0} m}{-p_{+}^{e} p_{-}^{p}-k_{\perp}^{2}-m^{2}+i \epsilon} \hat{\gamma}_{+} u\left(-p^{p}\right)+\bar{u}\left(p^{e}\right) \frac{-\hat{\vec{\alpha}}_{\perp} \cdot\left(-\vec{p}_{\perp}^{p}+\vec{p}_{\perp}^{e}-\vec{k}_{\perp}\right)+\gamma_{0} m}{-p_{-}^{e} p_{+}^{p}-\left(-\vec{p}_{\perp}^{p}+\vec{p}_{\perp}^{e}-k_{\perp}\right)^{2}-m^{2}+i \epsilon} \hat{\gamma}_{-} u\left(-p^{p}\right)\right) \\
& \times\left(\bar{u}\left(p^{e}\right) \frac{-\hat{\vec{\alpha}}_{\perp} \cdot \vec{k}_{\perp}^{\prime}+\gamma_{0} m}{-p_{+}^{e} p_{-}^{p}-k_{\perp}^{\prime 2}-m^{2}+i \epsilon} \hat{\gamma}_{+} u\left(-p^{p}\right)+\bar{u}\left(p^{e}\right) \frac{-\hat{\vec{\alpha}}_{\perp} \cdot\left(-\vec{p}_{\perp}^{p}+\vec{p}_{\perp}^{e}-\vec{k}_{\perp}^{\prime}\right)+\gamma_{0} m}{-p_{-}^{e} p_{+}^{p}-\left(-\vec{p}_{\perp}^{p}+\vec{p}_{\perp}^{e}-k_{\perp}^{\prime}\right)^{2}-m^{2}+i \epsilon} \hat{\gamma}_{-} u\left(-p^{p}\right)\right) \text {. }
\end{aligned}
$$

The integration over impact parameter yields the pair-production cross section. Due to the $\delta^{2}\left(\vec{k}_{\perp}^{\prime}-\vec{k}_{\perp}\right)$ function arising from the $\vec{b}$ integration, a further momentum integral can be performed, leaving

$$
\begin{aligned}
d \sigma= & \frac{m d^{3} p^{e}}{(2 \pi)^{3} E^{e}} \frac{m d^{3} p^{p}}{(2 \pi)^{3} E^{p}} \int \frac{d^{2} k_{\perp}}{(2 \pi)^{2}}\left|\mathcal{T}_{B}\left(\vec{p}_{\perp}^{e}-\vec{k}_{\perp}\right)\right|^{2}\left|\mathcal{T}_{A}\left(\vec{k}_{\perp}+\vec{p}_{\perp}^{p}\right)\right|^{2} \mid \vec{u}\left(p^{e}\right) \frac{-\hat{\vec{\alpha}}_{\perp} \cdot \vec{k}_{\perp}+\gamma_{0} m}{-p_{+}^{e} p_{-}^{p}-k_{\perp}^{2}-m^{2}+i \epsilon} \hat{\gamma}_{+} u\left(-p^{p}\right) \\
& +\left.\bar{u}\left(p^{e}\right) \frac{-\hat{\vec{\alpha}}_{\perp} \cdot\left(-\vec{p}_{\perp}^{p}+\vec{p}_{\perp}^{e}-\vec{k}_{\perp}\right)+\gamma_{0} m}{-p_{-}^{e} p_{+}^{p}-\left(-\vec{p}_{\perp}^{p}+\vec{p}_{\perp}^{e}-k_{\perp}\right)^{2}-m^{2}+i \epsilon} \hat{\gamma}_{-} u\left(-p^{p}\right)\right|^{2} .
\end{aligned}
$$

Thus, upon integration over the whole impact parameter plane, the phases that are present in the individual scattering amplitudes [see Eq. (15)] cancel. Consequently, in the limit $\gamma \rightarrow \infty$, the cross section is found to reduce to the lowestorder perturbation theory result, the two-photon graph. This behavior naturally explains [23] the experimentally observed quadratic dependence on the target's and projectile's charge [24]. It also implies that no asymmetries should occur in electron and positron spectra.

Equation (37) is strictly valid only for pointlike ions and it includes arbitrarily close collisions. The focus on electromagnetic reactions in peripheral heavy-ion collisions implies a restricted range of impact parameters with a lower bound $b=r_{A}+r_{B}, r_{A}$ and $r_{B}$ being the radii of the ions. Therefore, in experiments that are triggered on peripheral collisions, effects of the Coulomb distortions described by the phase in Eq. (15) should be visible.

The eikonal approximation (and thus the cross section) is known to become energy independent in the ultrarelativistic limit [25]. This dependence is restored by accounting for the correct transverse momentum range, which is restricted by the validity of Eq. (A8). This condition reads [26] (see Appendix A)

$$
\left|\vec{k}_{\perp}\right| \gg \frac{\omega}{\gamma}
$$

Such a low-momentum cutoff is also necessary to cure the IR divergence that arises from integrating over the poles of the momentum transfer functions $\mathcal{T}$ in Eqs. (30) or (37).

\section{EQUIVALENT-PHOTON APPROXIMATION}

In first-order perturbation theory the pair production probability, i.e., the impact parameter differential cross section, has been evaluated using analytical methods [27] or Monte Carlo integration [28]. Considering, however, the full interaction as in the previous section, the exact evaluation of Eq. (36) will require extensive numerical efforts, due to the oscillating phases contained in $\mathcal{T}$. To get a simple estimate of the solution, we intend to study the behavior of the cross section, both impact parameter dependent and impact parameter integrated, in the Weizsäcker-Williams method of equivalent photons. This approximation is based on the similarity between the fields of a fast moving charge and a swarm of real photons moving in beam direction. It approximately corresponds to the first-order Born approximation in the temporal gauge: Only the transverse part of the interaction is considered - the longitudinal part is suppressed by $1 / \gamma^{2}-$ and the vertex function is evaluated on shell at $k^{2}=0$, i.e., for an assumed real photon. Rewriting the exact cross section in terms of the real-photon cross section, the whole information about the scattering potential, which can be the retarded Coulomb potential or the modified potential (26), respectively, is then contained in the distribution function of the equivalent photons $n(\omega)$. Roughly speaking, this photon distribution function is determined by the squared absolute value of the Fourier-transformed potential (in temporal gauge). An obvious advantage of casting the exchange of effective photons according to Eq. (26) in the WeizsäckerWilliams form is that any difference between the secondorder perturbative result and the exact calculation will be solely generated by differences between the equivalent photon distributions. 
The Weizsäcker-Williams approximation is applicable if the exchanged momentum meets the following conditions [29]:

$$
\frac{\omega}{\gamma^{2}} \ll\left|\vec{k}_{\perp}\right| \ll m
$$

and

$$
\left|\vec{k}_{\perp}\right| \ll \omega \ll m \gamma .
$$

The upper bounds mainly stem from the requirement that $\left|k^{2}\right|$ is negligible compared to $2 p k \geqslant m^{2}$, such that the intermediate propagators of the scattered particles can be approximated by those describing the interaction with real photons. The particle's rest mass in Eq. (39) is, however, a conservative upper bound and the equivalent photon method is not strictly invalid for $\left|k^{2}\right| \sim m^{2}$. Note that for the approximate calculations in [10], the transverse mass $m_{\perp}$ of the scattered particle instead of $m$ was taken as the upper bound for Eq. (39).

Since the exact amplitude takes the eikonal form, we must emphasize the following: The expansion of the ultrarelativistic scattering amplitude in powers of the transferred momentum yields, as the leading term, the eikonal expression (describing the minimal deflection from the initial straightline trajectory) $[1,6]$. Its perturbation-theoretical derivation requires that the quadratic terms $k^{2}$ are negligible relative to the terms $2 p_{i} k$ in the denominators of the propagators, where $k$ is any partial sum of the internal momenta [7]. The exact validity of the eikonal formula, therefore, implies that at infinite scattering energies the transferred momentum $\left|k^{2}\right|$ is always smaller than $m^{2}$ (irrespective of the value of $m$ ). This agrees with the theoretical observation that at high energies particles are predominantly scattered into a cone with opening angle $\theta \sim 1 / \gamma$, corresponding to momentum transfers $\left|k^{2}\right| \sim m^{2}$. The main contributions to the cross section are thus expected from spatial distances larger or equal to the Compton wavelength of the particle. ${ }^{4}$

Moreover, the longitudinal part of the interaction vanishes identically in the limit $\gamma \rightarrow \infty$. Hence the applicability conditions of the Weizsäcker-Williams approximation are trivially fulfilled in the limit $\gamma \rightarrow \infty .^{5}$

We have the freedom to apply this method to the interaction of the electron with both nuclei, giving two possibilities (see Fig. 5). The two possible calculational schemes agree, since in (b) the bremsstrahlung emission and the scattering at the external potential decouple. This is due to the fact that the region in which the ultrarelativistic electron "feels" the external field is infinitely thin and any frequency of the emit-

\footnotetext{
${ }^{4}$ From the asymmetry of electron and positron spectra produced in $S(200 \mathrm{GeV} / n)+\mathrm{Au}$ collisions, the mean transverse distance from the target ion was deduced to be approximately two Compton wavelengths [24]. The collision energy corresponds to $\gamma \approx 10$ in the center-of-speed system.

${ }^{5}$ Just as the eikonal formula, the Weizsäcker-Williams approximation can be viewed as the leading term of an expansion in powers of $k^{2} / m^{2}$ [30]. The validity of the eikonal expression then automatically implies the validity of the Weizsäcker-Williams method.
}

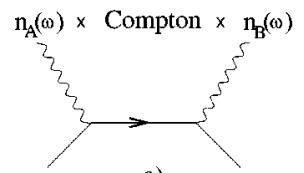

a)

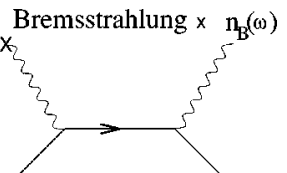

b)
FIG. 5. The two possible distinct processes that can be used to describe electron-positron production in heavy-ion collision. One or both ultrarelativistic ions can be replaced by an equivalent photon distribution. If the bremsstrahlung process (b) is calculated in one ion's rest frame, the electron must be assumed ultrarelativistic, to yield agreement with (a).

ted photon is "soft" compared to the time scale of the scattering. Coulomb effects arise, if one explicitly accounts for the finite interaction time, either in the scattering process by correcting the eikonal formula or by keeping the eikonal amplitude for the scattering process but assuming a Rutherforddeflected trajectory for the photon emission [29]. Corrections to the eikonal formula account for higher orders of, e.g., the Magnus expansion [15], which is an expansion in the interaction time $\tau$ around the instantaneous interaction $\tau \sim 1 / \gamma$ $\rightarrow 0$. In general these Coulomb effects vanish if the energy of the emitted photon is too small to resolve details of the scattering process, and the recoil of the electron is negligible.

To apply the Weizsäcker-Williams method to the bremsstrahlung photon, the recoil of this photon must, however, be assumed negligible. The small momentum transfer is in turn ensured by the eikonalization of the scattering process.

The equivalent single-photon distribution functions $n_{A / B}(\omega)$ of the ions $A$ and $B$ are given by the Poynting vector

$$
\begin{aligned}
n(\omega) & =\frac{1}{2 \pi \omega} \int d^{2} x_{\perp}|\vec{S}(\omega)| \\
& =\frac{1}{2 \pi \omega} \int d^{2} x_{\perp}\left|\operatorname{grad}_{\perp} A_{0}\left(\omega, \vec{x}_{\perp}\right)\right| .
\end{aligned}
$$

$A_{0}$ is the scalar part of the retarded Coulomb potential. For the case they are determined from the effective potential (26); they read

$$
\begin{aligned}
n(\omega) & =\frac{1}{4 \pi^{2} \alpha \omega} \int_{\omega / \gamma}^{m} k_{\perp} d k_{\perp}\left|k_{\perp} \pi Z \alpha\left(\frac{4}{k_{\perp}^{2}}\right)^{1-i Z \alpha} \frac{\Gamma(-i Z \alpha)}{\Gamma(i Z \alpha)}\right|^{2} \\
& =\frac{2 Z^{2} \alpha}{\pi \omega} \ln \left(\frac{m \gamma}{\omega}\right) .
\end{aligned}
$$

The lower bound of the integral is taken from the condition (38). The upper bound, the electron rest mass, is imposed by Eq. (39). The prefactor arises from properly rewriting the cross section (37) in terms of the real photon cross section (i.e., the Compton cross section) and photon distribution functions. 
The photon distribution (40) coincides with the equivalent-photon distribution obtained from the Coulomb potential to logarithmic accuracy [29] and is not changed by the higher-order effects.

\section{IMPACT-PARAMETER-DEPENDENT CROSS SECTION}

In [31] the impact-parameter-dependent equivalentphoton method has been developed. Using similar methods we can extend this treatment to the exact calculation, based on the modified potential (26). To this end we have to modify the integrands in Eq. (36) such that they account for the limited momentum range specified in Eqs. (38) and (39).

The cutoff of low transverse momenta according to Eq. (38) can be achieved by the following replacement in Eq. $(15):^{6}$

$$
t=-\vec{k}_{\perp}^{2} \rightarrow-\frac{\omega^{2}}{\gamma^{2}}-\vec{k}_{\perp}^{2}
$$

This substitution suppresses small transverse momenta less strongly than a strict cutoff at $k_{\perp}=\omega / \gamma$. It assumes the sufficient accuracy of the eikonal amplitude for $1 / \gamma$ in the near vicinity of $1 / \gamma=0$, which is guaranteed by the possibility to continuously extend the eikonal formula towards finite $\gamma$ and large $t$.

We briefly comment on a possible alternative cutoff procedure implemented in coordinate space. One might introduce a Yukawa-type screening of large transverse distances achieved by damping the modified potential (26) with the factor $e^{-\epsilon x_{\perp}}$. In accordance with the $k_{\perp}$ cutoff, the parameter $\epsilon$ can be set to the value $\omega / \gamma$; see [26] for a similar regularization. In momentum space this leads to the effective potential

$$
\begin{aligned}
A(k)= & (2 \pi)^{2} \delta\left(k_{-}\right) \frac{1}{\left(\epsilon^{2}+k_{\perp}^{2}\right)^{(1-i \alpha Z)}} \Gamma(2(1-i \alpha Z)) \\
& \times P_{1-2 i \alpha Z}\left[\epsilon\left(\epsilon^{2}+k_{\perp}^{2}\right)^{-1 / 2}\right]
\end{aligned}
$$

where $P_{1-2 i \alpha Z}$ denotes a Legendre function. This expression resembles the propagator of a photon with mass $\epsilon$. However, the last two factors in Eq. (42) change the character of the amplitude significantly and cannot be motivated physically.

In the ultraviolet region, in accordance with the exact validity of the eikonal formula at ultrarelativiastic energies, the transferred momenta are restricted by the condition $\left|k^{2}\right|$ $\ll m^{2}$. They are, however, in any case cut off naturally, if one introduces a form factor to account for the finite extension of the nuclei. Thus, large momenta have to be cut off at $k_{\perp} \approx 1 / r_{>}$, where $r_{>}$is the larger value of either the nuclear radius or the Compton wavelength of the scattered particle

\footnotetext{
${ }^{6}$ Note that for the Schrödinger case, the exact validity of the eikonal formula can be proven for a certain off-shell domain of the momentum transfer for the whole energy plane [32].
}

[33]. In this respect the electron is an exception, since all other particles have Compton wavelengths smaller or comparable to the nuclear size. To present the calculations in a uniform manner, we use the form factor of the nucleus to suppress large momenta.

The impact-parameter-dependent cross section for particle production, described in the equivalent photon method, reads [31]

$$
\begin{aligned}
\frac{d \sigma}{d^{2} b}= & \int d \omega_{1} \int d \omega_{2}\left[n_{\|}\left(\omega_{1}, \omega_{2}, \vec{b}\right) \sigma_{\|}^{\gamma \gamma}\left(\omega_{1}, \omega_{2}\right)\right. \\
& \left.+n_{\perp}\left(\omega_{1}, \omega_{2}, \vec{b}\right) \sigma_{\perp}^{\gamma \gamma}\left(\omega_{1}, \omega_{2}\right)\right]
\end{aligned}
$$

with the two-photon distribution functions $n_{\| / \perp}\left(\omega_{1}, \omega_{2}, \vec{b}\right)$. The elementary two-photon cross sections and the twophoton distribution functions explicitly account for the parallel or orthogonal orientation of the photon polarizations denoted by the indices $\|$ and $\perp$, respectively. Since we did not integrate over the impact parameter plane, which would have implied the averaging over the photon polarizations, the explicit occurrence of the photon polarizations in the impactparameter-dependent cross section is expected. The functions $n_{\| / \perp}\left(\omega_{1}, \omega_{2}, \vec{b}\right)$ can be expressed in terms of single-photon distribution functions $n(\omega, b)$, depending on the transverse separation:

$$
\begin{aligned}
n_{\|}\left(\omega_{1}, \omega_{2}, \vec{b}\right)= & \int d^{2} x_{\perp} n\left(\omega_{1}, \vec{x}_{\perp}-\vec{b}\right) n\left(\omega_{2}, \vec{x}_{\perp}\right) \\
& \times\left(\frac{\left(\vec{x}_{\perp}-\vec{b}\right) \cdot \vec{x}_{\perp}}{\left|\vec{x}_{\perp}-\vec{b}\right|\left|\vec{x}_{\perp}\right|}\right)^{2}, \\
n_{\perp}\left(\omega_{1}, \omega_{2}, \vec{b}\right)= & \int d^{2} x_{\perp} n\left(\omega_{1}, \vec{x}_{\perp}-\vec{b}\right) n\left(\omega_{2}, \vec{x}_{\perp}\right) \\
& \times\left(\frac{\left(\vec{x}_{\perp}-\vec{b}\right) \times \vec{x}_{\perp}}{\left|\vec{x}_{\perp}-\vec{b}\right|\left|\vec{x}_{\perp}\right|}\right)^{2}
\end{aligned}
$$

with

$$
n\left(\omega, x_{\perp}\right)=\frac{Z^{2} \alpha}{\pi^{2} \omega}\left|\int_{0}^{\infty} d k_{\perp} k_{\perp}^{2} \frac{F\left(k_{\perp}^{2}+\omega^{2} / \gamma^{2}\right)}{\left(k_{\perp}^{2}+\omega^{2} / \gamma^{2}\right)^{1-i Z \alpha}} J_{1}\left(x_{\perp} k_{\perp}\right)\right|^{2} .
$$

$J_{1}$ is a Bessel function. The function $F$ denotes the charge form factor of the nucleus.

For a pointlike charge $(F \equiv 1)$, the photon distribution function can be calculated analytically. For the Coulomb potential and the modified potential we obtain 


$$
n\left(\omega, x_{\perp}\right)= \begin{cases}\frac{Z^{2} \alpha}{\pi^{2}} \frac{\omega}{\gamma^{2}}\left[K_{1}\left(\frac{\omega x_{\perp}}{\gamma}\right)\right]^{2} & \text { retarded Coulomb potential } \\ \frac{Z^{2} \alpha}{\pi^{2}} \frac{\omega}{\gamma^{2}}\left|\frac{K_{1+i Z \alpha}\left(\omega x_{\perp} / \gamma\right)}{\Gamma(1-i Z \alpha)}\right|^{2} & \text { modified potential (26). }\end{cases}
$$

$K_{\nu}$ is a modified Bessel function. For small arguments of the Bessel function one can use the asymptotic expression [34]

$$
K_{\nu}(z) \sim \frac{1}{2} \Gamma(\nu)\left(\frac{1}{2} z\right)^{-\nu}
$$

Therefore, for $\omega b \ll \gamma$ and assuming pointlike charges, the photon distribution functions (47) nearly completely agree.

We have numerically evaluated the photon distribution function (46) for an extended nucleus, using a Gaussian form factor $F\left(Q^{2}\right)=e^{-Q^{2} /\left(2 Q_{0}^{2}\right)}$ with $Q_{0}=60 \mathrm{MeV}$, which approximately describes a $\mathrm{Pb}$ nucleus [35]. Figure 6 shows a comparison between the photon distribution functions for both, pointlike nuclei $(F \equiv 1)$ and extended nuclei using either the retarded Coulomb potential or the modified potential (26), which represents the exact calculation. At small distances up to a few multiples of the nuclear radius, the modified potential gives a smaller number of equivalent photons than the pure Coulomb potential. For large distances or large photon energies, Eq. (48) loses its validity and the modified potential gives a larger number of photons than the Coulomb potential (see Fig. 7). According to Eq. (47) the ratio of the photon numbers generated of the Coulomb potential and the modified potential at large arguments is given by

$$
\frac{n_{C}\left(\omega, x_{\perp}\right)}{n_{m o d}\left(\omega, x_{\perp}\right)} \rightarrow|\Gamma(1-i Z \alpha)|^{2}=\frac{\pi Z \alpha}{\sinh (\pi Z \alpha)}
$$

The impact-parameter-dependent two-photon distribution functions have to be corrected according to Eq. (46), thus correcting the pair production probability (43) for Coulomb

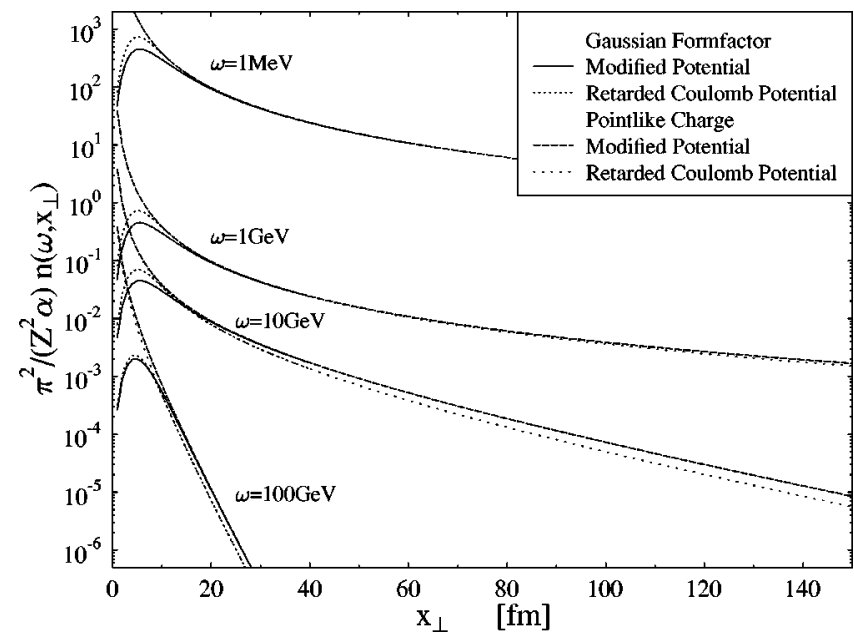

FIG. 6. The single-photon distribution function for various photon energies (indicated in the plot) as a function of the transverse distance from the ultrarelativistic charge. The calculations are done for lead ions $(Z=82)$ at LHC energies $(\gamma=3000)$. effects. Due to the complicated convolution of the singlephoton distribution functions in Eqs. (44) and (45), effects on the two-photon distribution functions are not obvious. For large distances $x_{\perp} \gg b$, however, one directly finds an enhancement of the equivalent two-photon numbers.

\section{SUMMARY}

In this paper we have investigated the scattering of Dirac particles in the field of one or two moving charges. We have found that the eikonal expression for the scattering amplitude becomes exact in the ultrarelativistic limit $(\gamma \rightarrow \infty)$. This was shown by either summing the perturbation series or by matching plane waves at the delta-function potential on the light front (see also [9,26]), using the transformation presented in Sec. II A. At ultrarelativistic energies one can neglect the squared momentum transfer $k^{2}$ relative to the term $2 p_{i} k$ in the denominator of the propagator of the scattered particle. As a consequence, the applicability conditions of the Weizsäcker-Williams method are fulfilled automatically -irrespective of the mass of the scattered particle.

Furthermore, the exact validity of the eikonal formula for ultrarelativistic scattering processes confirms the Pomeranchuk theorem, stating that the cross sections for antiparticle and particle scattering at a given target become identical in the ultrarelativistic limit. One can describe the exact interaction as the exchange of an effective photon, according to a

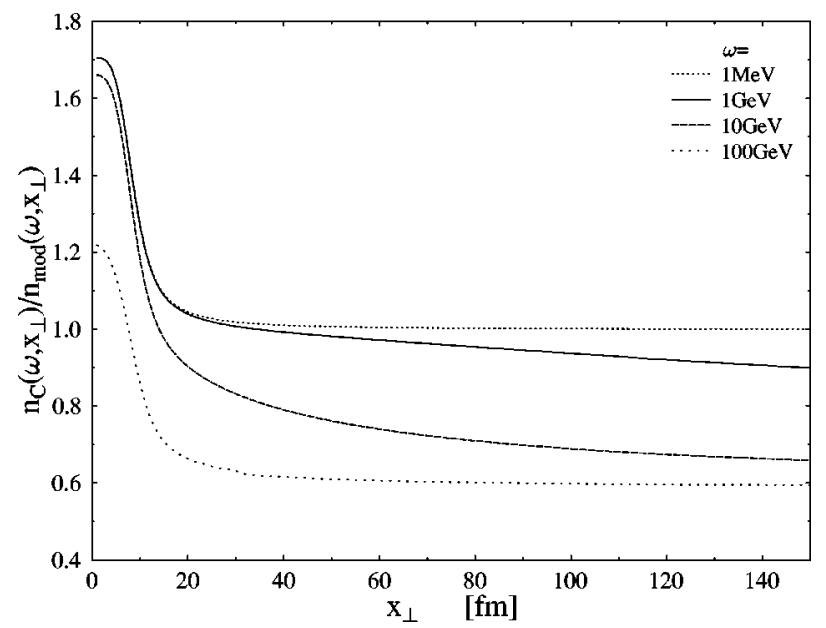

FIG. 7. The ratio of the equivalent photon numbers of the pure Coulomb potential, $n_{C}\left(\omega, x_{\perp}\right)$, and the photon numbers of the modified potential, $n_{\text {mod }}\left(\omega, x_{\perp}\right)$, calculated for a lead nucleus $(Z$ $=82$ ). At small transverse distances, one finds a deviation of up to $70 \%$ for small photon energies. Far outside the nucleus, the photon distribution functions are given by Eq. (47). For large distances, i.e., large arguments in Eq. (47), one asymptotically finds $n_{C} / n_{\text {mod }}$ $\rightarrow 0.578$ [see Eq. (49)]. 
modified, effective potential. The cross section, as a peculiarity of the Coulomb interaction, becomes identical to the Mott result. Due to the discussed causal decoupling property, the exact pair production amplitude in the field of two ultrarelativistic colliding (pointlike) nuclei was shown to retain the structure of the second-order perturbative result. It can, therefore, be treated like a two-photon process with a modified potential. As in the case of single scattering, the cross section reduces to the second-order perturbative result [26], which was evaluated in [10]. This allows for two conclusions: (i) The production rate scales with the square of the target and the projectile charge $[24,23]$. (ii) Asymmetries in the electron and positron spectra should not occur.

Note, however, that the presented formalism is valid only if the produced particles are fast with respect to both nuclei. Therefore, the observed [24] asymmetry at small electron and positron momenta remains unaffected by these considerations.

We applied the Weizsäcker-Williams approach to pair production using the modified potential (26), which correctly accounts for the Coulomb boundary conditions. In this way we derive an equivalent-photon distribution, which directly reveals the effects of Coulomb distortions. The impactparameter-dependent single-photon distribution calculated with the modified potential shows deviations from the equivalent photon distribution function obtained from the retarded Coulomb potential. For a lead nucleus at (LHC) energies we find deviations up to $70 \%$ at small separations and approximately $40 \%$ at large separations from the ion.

The pair-production probability is subject to changes due to the modified photon numbers at given impact parameters and photon energies. The perturbation theoretical probability, as calculated here, rather represents the average number of produced pairs and exceeds unity at sufficiently small impact parameters. The "true" pair-production probability has to be corrected by the vacuum-to-vacuum amplitude, which in turn can be calculated from the perturbative pairproduction probability $[36,37]$. This nontrivial influence of Coulomb effects on the pair-production cross section is the subject of further studies.

\section{ACKNOWLEDGMENTS}

U.E. would like to thank S.J. Chang for helpful comments, and D. Schwarz and F. Constantinescu for stimulating discussions. This work was supported by the Deutsche Forschungsgemeinschaft (DFG) within Project No. Gr-243/44-2.

\section{APPENDIX A: ULTRARELATIVISTIC LIMIT OF THE POTENTIAL}

In this section we want to discuss the limit $\beta \rightarrow 1$ of the potential (1). From Sec. II A we expect the asymptotic form of the potential to be

$$
\lim _{\beta \rightarrow 1} \frac{-1}{\sqrt{(z-t)^{2}+\frac{\vec{x}_{\perp}^{2}}{\gamma^{2}}}}=\delta(z-t) \ln \left(x_{\perp}^{2}\right)+\mathcal{C}^{\prime} .
$$

As motivated in Sec. II A, $\mathcal{C}$ is determined by the principal value of the infinite time integral, so that $\mathcal{C}^{\prime}$ can be inferred to be

$$
\mathcal{C}^{\prime}=\frac{-1}{|z-t|}-\delta(z-t) \ln \left(\gamma^{2}\right),
$$

where we had to require $\gamma \gg x_{\perp} /|z-\beta t|$.

An attempt to derive the limit by means of Fourier transformation of Eq. (1) with respect to $z$ was presented in [3]. The Fourier transform reads

$$
\begin{aligned}
\int d z e^{i \omega z} \frac{1}{\sqrt{(z-\beta t)^{2}+\frac{\vec{x}_{\perp}^{2}}{\gamma^{2}}}} & =e^{i \omega \beta t} \int d z e^{i \omega z} \frac{1}{\sqrt{z^{2}+\frac{\vec{x}_{\perp}^{2}}{\gamma^{2}}}} \\
& \rightarrow-2 e^{i \omega t} \ln \left(\frac{\omega\left|\vec{x}_{\perp}\right| e^{C}}{2 \gamma}\right) .
\end{aligned}
$$

The quantity $C$ here denotes Euler's constant. Naive application of the textbook formula [38] for the inverse Fourier transformation of this expression

$$
\int \frac{d k}{2 \pi} \ln |k| e^{i k x}=-\frac{1}{2|x|}
$$

would give

$$
\lim _{\beta \rightarrow 1} \frac{1}{\sqrt{(z-t)^{2}+\frac{\vec{x}_{\perp}^{2}}{\gamma^{2}}}}=\frac{1}{|z-t|} .
$$

Equation (A4) is, however, valid only up to arbitrary multiples of $\delta(x)$. The validity of Eq. (A3), however, as well demands the condition $\gamma \gg \omega x_{\perp}$.

It is possible to find a gauge transformation that removes both the long-range potential $1 /|z-t|$ as well as $\delta(z$ $-t) \ln \left(\gamma^{2}\right)$, see Eq. (A2). This is achieved by the gauge transformation [13]

$$
\psi^{\prime}=e^{i Z \alpha \ln \left[\gamma(z-\beta t)+\sqrt{1+\gamma^{2}(z-\beta t)^{2}}\right]} \psi .
$$

The gauge-transformed potential reads

$$
A_{0}^{\prime}=-\frac{Z \alpha \gamma}{\sqrt{\gamma^{2}(z-\beta t)^{2}+\vec{x}_{\perp}^{2}}}+\frac{Z \alpha \gamma}{\sqrt{\gamma^{2}(z-\beta t)^{2}+1}}
$$

and has the ultrarelativistic limit $[13,39]$

$$
\lim _{\beta \rightarrow 1} A_{0}^{\prime}=Z \alpha \delta(z-t) \ln \left(\vec{x}_{\perp}^{2}\right) .
$$

The appearance of the logarithm follows immediately from the inhomogeneous Maxwell equation in the Lorentz gauge, which reduces to a two-dimensional Poisson equation in the limit $\beta \rightarrow 1$. 
The gauge transformation (A6) has the advantage to yield a short-range potential that allows for asymptotic plane-wave solutions. For this reason it was used to obtain a faster convergence in coupled channel calculations [40].

\section{APPENDIX B: SOLUTION OF THE DIRAC EQUATION IN THE FIELD OF COLLIDING IONS}

The Dirac equation for an electron moving in the field of two ultrarelativistic colliding ions $A$ and $B$ reads

$$
\begin{aligned}
& {\left[\frac{1}{2}\left(\hat{\gamma}_{-} i \partial_{\tau_{-}}+\hat{\gamma}_{+} i \partial_{\tau_{+}}\right)+i \hat{\vec{\gamma}}_{\perp} \cdot \vec{\nabla}_{\perp}-m-\frac{1}{2} \hat{\gamma}_{-} \delta\left(\tau_{-}\right) V_{\perp}^{A}\right.} \\
& \left.\quad-\frac{1}{2} \hat{\gamma}_{+} \delta\left(\tau_{+}\right) V_{\perp}^{B}\right] \psi=0
\end{aligned}
$$

where we used light cone variables $\tau_{ \pm}=(t \pm z) / 2$. One directly finds that $\hat{\gamma}_{-} \psi$ is discontinuous at $\tau_{-}$through the action of ion $A$ and $\hat{\gamma}_{+} \psi$ is discontinuous at $\tau_{+}=0$ through the action of ion $B$, respectively.

We introduce $\psi_{ \pm}=\left(1 \pm \hat{\alpha}_{z}\right) \psi$ and use $2 \psi=\psi_{-}+\psi_{+}$to formulate the problem as follows:

$$
\begin{aligned}
& {\left[i \partial_{\tau_{+}}-\delta\left(\tau_{+}\right) V_{\perp}^{B}\right] \psi_{+}+\left(i \hat{\vec{\alpha}}_{\perp} \cdot \vec{\nabla}_{\perp}-\gamma_{0} m\right) \psi_{-}} \\
& \quad=-\left[i \partial_{\tau_{-}}-\delta\left(\tau_{-}\right) V_{\perp}^{A}\right] \psi_{-}-\left(i \hat{\vec{\alpha}}_{\perp} \cdot \vec{\nabla}_{\perp}-\gamma_{0} m\right) \psi_{+},
\end{aligned}
$$

where Eq. (B1) has been multiplied by $2 \hat{\gamma}_{0}$. By using the standard representation of Dirac matrices and rearranging the four equations (B2), one obtains

$$
\begin{aligned}
& {\left[\left(\begin{array}{ll}
1 & 0 \\
0 & 0
\end{array}\right)\left(i \partial_{\tau_{+}}-\delta\left(\tau_{+}\right) V_{\perp}^{B}\right)+\left(\begin{array}{ll}
0 & 0 \\
0 & 1
\end{array}\right)\left(i \partial_{\tau_{-}}-\delta\left(\tau_{-}\right) V_{\perp}^{A}\right)\right.} \\
& -m\left(\begin{array}{ll}
0 & 1 \\
1 & 0
\end{array}\right)+i \partial_{x}\left(\begin{array}{rr}
0 & -\sigma_{y} \\
\sigma_{y} & 0
\end{array}\right) \\
& \left.+i \partial_{y}\left(\begin{array}{rr}
0 & -i \sigma_{x} \\
i \sigma_{x} & 0
\end{array}\right)\right] \widetilde{\psi}=0,
\end{aligned}
$$

where

$$
\widetilde{\psi}=\left(\begin{array}{c}
\psi_{1}+\psi_{3} \\
\psi_{2}-\psi_{4} \\
\psi_{1}-\psi_{3} \\
\psi_{2}+\psi_{4}
\end{array}\right),
$$

corresponding to an isomorphic linear transformation [9] with the matrix

$$
\Lambda=\left(\begin{array}{cc}
1 & \sigma_{z} \\
1 & -\sigma_{z}
\end{array}\right)
$$

Since $\Lambda$ is a bijection, each side of Eq. (B2) has to be zero. Off the light fronts we, therefore, have the two equations

$$
i \partial_{\tau_{+}} \psi_{+}=\left(i \hat{\vec{\alpha}}_{\perp} \cdot \vec{\nabla}_{\perp}-\gamma_{0} m\right) \psi_{-},
$$

$$
i \partial_{\tau_{-}} \psi_{-}=\left(i \hat{\vec{\alpha}}_{\perp} \cdot \vec{\nabla}_{\perp}-\gamma_{0} m\right) \psi_{+}
$$

According to Eq. (12) the discontinuities at the light fronts are described by

$$
\begin{aligned}
& \psi_{-}\left(\tau_{-}=0^{+}\right)=\phi^{A}\left(x_{\perp}\right) \psi_{-}\left(\tau_{-}=0^{-}\right), \\
& \psi_{-}\left(\tau_{+}=0^{+}\right)=\phi^{B}\left(x_{\perp}\right) \psi_{+}\left(\tau_{-}=0^{-}\right) .
\end{aligned}
$$

$\phi^{A}$ and $\phi^{B}$ are defined by Eq. (6) using the scalar parts of the potentials of the ions $A$ and $B$. Let us study the spinor $\psi_{+}$, evaluated at the surface $\tau_{+}=0^{+}$:

$$
\psi_{+}\left(\tau_{+}=0^{+}\right)=\phi^{B} \frac{i \hat{\vec{\alpha}}_{\perp} \cdot \vec{\nabla}_{\perp}-\gamma_{0} m}{p_{-}} \psi_{-}\left(\tau_{+}=0^{-}\right) .
$$

In the region $\tau_{-}>0$ the electron already has interacted with ion $A$ and we can write

$$
\psi_{+}\left(\tau_{+}=0^{+}, \tau_{-}>0\right)=\phi^{B} \phi^{A} \frac{i \hat{\overrightarrow{\alpha_{\perp}}} \cdot \vec{\nabla}_{\perp}-\gamma_{0} m}{p_{-}}\left(1-\alpha_{z}\right) \psi_{p}
$$

where $\psi_{p}$ is the incoming plane wave with momentum $p$. This relation also can be obtained immediately from Eqs. (28) and (B5) for $\tau_{+}$and $\tau_{-}>0$. The operator $i \partial_{+}$in Eq. (B5) has been replaced by its eigenvalue $p_{-}$, the incoming negative light cone momentum. This is possible since $p_{-}$is conserved in the interaction with ion $A$. The expansion of $\psi_{+}\left(\tau_{+}=0^{+}\right)$in the plane-wave basis reads

$$
\begin{aligned}
\psi_{+}\left(\tau_{+}=0^{+}, \tau_{-}>0\right) \\
\quad=\int \frac{d p_{+} d^{2} p_{\perp}}{(2 \pi)^{3}} B\left(p^{\prime}, p\right) e^{-i p_{+} \tau_{-}+i \vec{p}_{\perp} \cdot \vec{x}_{\perp}} \sqrt{\frac{m}{p_{0}}} u(p),
\end{aligned}
$$

where we substituted $d^{3} p^{\prime} \rightarrow d p_{+}^{\prime} d^{2} p_{\perp}^{\prime}$ [16]. According to Eq. (B7) the expansion coefficients are

$$
\begin{gathered}
B\left(p^{\prime}, p\right) \sqrt{\frac{m}{p_{0}}} u(p)=\int_{0}^{\infty} d \tau_{-} \int d^{2} x_{\perp} e^{i p_{+}^{\prime} \tau_{-}-i \vec{p}^{\prime}} \cdot_{\perp} \vec{x}_{\perp} \phi^{B} \\
\times \frac{i \hat{\vec{\alpha}}_{\perp} \cdot \vec{\nabla}_{\perp}-\gamma_{0} m}{p_{-}} \psi_{-}\left(\tau_{+}=0^{-}, \tau_{-}>0\right) .
\end{gathered}
$$

In the region $\tau_{-}>0, \tau_{+}<0$ the wave function $\psi_{-}$is a freely propagating wave packet with a fixed light cone momentum $p_{-}$and a superposition of transverse momenta $\vec{p}_{\perp}$. The mass-shell condition requires $p_{-} p_{+}=\vec{q}_{\perp}^{2}+m^{2}$. In this way $\psi_{-}\left(\tau_{+}<0, \tau_{-}>0\right)$ can be obtained from $\psi_{-}\left(\tau_{+}<0, \tau_{-}\right.$ $\left.=0^{+}\right)$. We have 


$$
\begin{aligned}
\psi_{-}\left(\tau_{-}>0\right)= & \int \frac{d^{2} q_{\perp}}{(2 \pi)^{2}} \exp \left(-i \frac{q_{\perp}^{2}+m^{2}}{p_{-}} \tau_{-}-i p_{-} \tau_{+}\right. \\
& \left.+i \vec{q}_{\perp} \cdot \vec{x}_{\perp}\right) \int d^{2} x_{\perp}^{\prime} e^{i \vec{x}_{\perp}^{\prime} \cdot\left(\vec{p}_{\perp}-\vec{q}_{\perp}\right)} \phi^{A} \\
& \times\left(1-\hat{\alpha}_{z}\right) \sqrt{\frac{m}{p_{0}}} u(p),
\end{aligned}
$$

which leads to

$$
\begin{aligned}
& \sqrt{\frac{m}{p_{0}^{\prime}}} u^{\dagger}\left(p^{\prime}\right) B\left(p^{\prime}, p\right) \sqrt{\frac{m}{p_{0}}} u(p) \\
& =-i \int \frac{d^{2} q_{\perp}}{(2 \pi)^{2}} \int d^{2} x_{\perp}^{\prime} e^{i \vec{x}_{\perp}^{\prime} \cdot\left(\vec{q}_{\perp}-\vec{p}_{\perp}^{\prime}\right)} \phi^{B} \\
& \times \int d^{2} x_{\perp} e^{i \vec{x}_{\perp} \cdot\left(\vec{p}_{\perp}-\vec{q}_{\perp}\right)} \phi^{A} \frac{m}{\sqrt{p_{0}^{\prime} p_{0}}} u^{\dagger}\left(p^{\prime}\right) \\
& \times \frac{\hat{\vec{\alpha}}_{\perp} \cdot \vec{q}_{\perp}+\gamma_{0} m}{p_{+}^{\prime} p_{-}-q_{\perp}^{2}-m^{2}+i \epsilon}\left(1-\hat{\alpha}_{z}\right) u(p) .
\end{aligned}
$$

Note that the lower boundary of the $\tau_{-}$integration is 0 , since we inserted the expression of $\psi_{-}$for $\tau_{-}>0$.

Together with the corresponding term for the reverse order of interactions with the two ions, Eq. (B12) is the $S$ matrix for an electron scattered at the light fronts, first derived by Segev and Wells [9] in an elegant way using the transformation Eq. (B4).

\section{APPENDIX C: SOLUTION IN THE FIELD OF CHANNELED IONS}

The causal decoupling of interactions with sources moving on the positive and negative light cone, respectively, and the fact that the derivations are valid for arbitrary transverse parts of the potential, can be used to calculate the electronpositron pair production for a field configuration, which corresponds to the channeling of an ion in a crystal.

We use the equal speed system; the crystal is moving in the $-z$ direction. The crystal layers have a spatial distance $a \vec{e}_{z}$. In the ultrarelativistic case, the electron again interacts with the ion and the crystal layers separately and we get simple time orderings of the interaction. For the sake of simplicity we formulate the perturbative description of the successive interactions of the electron with both the ion and the crystal layers directly with modified potentials of the form (26). One then obtains for the interaction with two neighboring crystal layers the integral

$$
\begin{aligned}
& S\left(p^{\prime} p\right)=-i \delta\left(p_{+}^{\prime}-p_{+}\right) e^{i p_{-}^{\prime} a / 2} \\
& \times \int \frac{d k_{-} d^{2} k_{\perp}}{(2 \pi)^{2}} \frac{\bar{u}\left(p^{\prime}\right) \hat{\gamma}_{+} u(p)}{k_{-}-\frac{k_{\perp}^{2}+m^{2}}{k_{+}}+\frac{i \epsilon}{k_{+}}} \\
& \times e^{-i k_{-} a / 2} F_{p^{\prime} k}\left(e^{-i V_{\perp}^{C_{i+1}}\left(\vec{x}_{\perp}\right)}\right) F_{k p}\left(e^{-i V_{\perp}^{C_{i}}\left(\vec{x}_{\perp}\right)}\right) \\
& =2 \pi \delta\left(p_{+}^{\prime}-p_{+}\right) \int \frac{d^{2} k_{\perp}}{(2 \pi)^{2}} \exp \left[i \left(-\frac{k_{\perp}^{2}+m^{2}}{2 p_{+}^{\prime}}\right.\right. \\
& \left.\left.+\frac{p_{-}^{\prime}}{2}\right) a\right] F_{p^{\prime} k}\left(e^{-i V_{\perp}^{C_{i+1}}(\vec{x})} F_{k p}\left(e^{-i V_{\perp}^{C_{i}}\left(\vec{x}_{\perp}\right)}\right)\right. \\
& \times \bar{u}\left(p^{\prime}\right) \hat{\gamma}_{+} u(p), \quad a>0, \quad \epsilon \rightarrow 0
\end{aligned}
$$

Here $V_{\perp}^{C_{i+1}}\left(\vec{x}_{\perp}\right)$ denotes the eikonal for the interaction of the electron with the $i$ th crystal layer, which will contain a superposition of atomic scattering centers. For $a<0$ (reverse direction of electron motion) the integral vanishes, which expresses that the electron cannot interact alternately with neighboring crystal layers, due to causality.

If the electron interacts with the channeled ion between interacting with two distinct crystal layers, we get

$$
\begin{aligned}
& S\left(p^{\prime} p\right)=\int \frac{d^{2} k_{\perp} d^{2} k_{\perp}^{\prime}}{(2 \pi)^{4}} \frac{\exp \left(i p_{-}^{\prime} a / 2\right)\left\{\exp \left[-i\left(k_{\perp}^{2}+m^{2}\right) a /\left(2 p_{+}\right)\right]-\exp \left[-i\left(k_{\perp}^{\prime 2}+m^{2}\right) a /\left(2 p_{+}^{\prime}\right)\right]\right\}}{p_{+}^{\prime}\left(k_{\perp}^{2}+m^{2}\right)-p_{+}\left(k_{\perp}^{\prime 2}+m^{2}\right)-i \epsilon\left(p_{+}^{\prime}-p_{+}\right)} F_{p^{\prime} k^{\prime}}\left[e^{-i V_{\perp}^{C_{i+1}}\left(\vec{x}_{\perp}\right)}\right]
\end{aligned}
$$

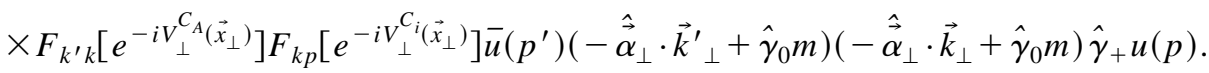


Successive interactions with different crystal layers factorize and any scattering process including intermediate interaction with the channeled ion gives the same amplitude.

Using the functional relation (28) directly gives the above perturbative results: If both ions $A$ and $B$ move on positive light cones separated by the spatial distance $a \vec{e}_{z}$, we obtain for the interacting part of the spinor $\psi$,

$$
\begin{aligned}
& \psi_{+}\left(\tau_{+}-a / 2=0^{+}\right) \\
& =\phi^{A} \psi_{+}\left(\tau_{+}-a / 2=0^{-}\right) \\
& =\phi^{A} \int \frac{d^{2} q_{\perp}}{(2 \pi)^{2}} \exp \left(-i \frac{q_{\perp}^{2}+m^{2}}{p_{+}} \frac{a}{2}-i p_{+} \tau_{-}+i \vec{q}_{\perp} \cdot \vec{x}_{\perp}\right) \\
& \times \int d^{2} x_{\perp}^{\prime} e^{i \overrightarrow{x^{\prime}} \perp \cdot\left(\vec{p}_{\perp}-\vec{q}_{\perp}\right)} \phi^{B}\left(1+\hat{\alpha}_{z}\right) \sqrt{\frac{m}{p_{0}}} u(p) .
\end{aligned}
$$

The expansion of $\psi_{+}$in plane waves at the point $\tau_{+}=a / 2$ $+0^{+}$yields the $S$ matrix of this process in momentum space

$$
\begin{aligned}
& S\left(p^{\prime}, p\right)=2 \pi i \delta\left(p_{+}^{\prime}-p_{+}\right) \int \frac{d^{2} q_{\perp}}{(2 \pi)^{2}} \\
& \times \exp \left[i\left(-\frac{q_{\perp}^{2}+m^{2}}{2 p_{+}^{\prime}}+\frac{p_{-}^{\prime}}{2}\right) a\right] \\
& \times \int d^{2} x_{\perp} e^{i \overrightarrow{x_{\perp}} \cdot\left(\vec{q}_{\perp}-\vec{p}^{\prime}{ }_{\perp}\right)} \phi^{A} \\
& \times \int d^{2} x_{\perp} e^{i \vec{x}_{\perp} \cdot\left(\vec{p}_{\perp}-\vec{q}_{\perp}\right)} \phi^{B} \frac{m}{\sqrt{p_{0}^{\prime} p_{0}}} \\
& \times u^{\dagger}\left(p^{\prime}\right)\left(1+\hat{\alpha}_{z}\right) u(p)
\end{aligned}
$$

in accordance with Eq. (C1).

Further studies will have to show how these considerations can be put to use for the calculation of pair creation in channeling.

\section{APPENDIX D: THE PHOTON PROPAGATOR AT HIGH COLLISION ENERGIES}

The four-dimensional Fourier transform of the retarded potential (1) reads

$$
\begin{aligned}
A_{0}(k) & =\int d^{4} x e^{i k x} \frac{-Z \alpha \gamma}{\sqrt{\gamma^{2}(z-\beta t)^{2}+\vec{x}_{\perp}^{2}}} \\
& =-(2 \pi)^{2} Z \alpha \delta\left(k_{0}-\beta k_{3}\right) \frac{2}{\left(\frac{k_{3}}{\gamma}\right)^{2}+\vec{k}_{\perp}^{2}},
\end{aligned}
$$

which has the following low- and high-velocity limits:

$$
\lim _{\beta \rightarrow 0} A_{0}(k)=-(2 \pi)^{2} Z \alpha \delta\left(k_{0}\right) \frac{2}{|\vec{k}|^{2}},
$$

$$
\lim _{\beta \rightarrow 1} A_{0}(k)=-(2 \pi)^{2} Z \alpha \delta\left(k_{0}-k_{3}\right) \frac{2}{\vec{k}_{\perp}^{2}} .
$$

The last expression reflects the observation that in the high-energy limit the longitudinal components $k_{-}$and $k_{+}$of the photon momentum can be dropped.

After having performed the gauge transformation (A6) and taken the limit $\gamma \rightarrow \infty$, the potential to be transformed is expression (A8). Grignani and Mintchev [20] have shown that it is wrong to identify the Fourier transform of Eq. (A8) with Eq. (D3) or with the regulated expression $1 /\left(\vec{k}_{\perp}^{2}+\mu^{2}\right)$ with a regulating mass inserted by hand.

Calculating the time integral of $A_{0}$ in the exponent of the eikonal expression, using Eq. (D1), and inserting a photon mass $\mu$ as above, one finds

$$
\int_{-\infty}^{\infty} d t A_{0}=Z \alpha \ln \left(\vec{x}_{\perp}^{2}\right)+Z \alpha \underset{\mu \rightarrow 0}{\lim \ln } \mu^{2}
$$

with $1 / 2 e^{C}$ absorbed in $\mu$ as in [3]. The term $\lim _{\mu \rightarrow 0} Z \alpha \ln \mu^{2}$ is the divergent constant $\mathcal{C}$ introduced in Sec. II A. It is interesting to note that this introduction of an infrared regulating photon mass does not yield a regularized expression for the eikonal amplitude.

One may attempt to calculate the two-dimensional Fourier transform of Eq. (A8) from a Taylor expansion in powers of $i Z \alpha$ of the momentum transfer function $\mathcal{T}(23)$, which for $V_{\perp}\left(\vec{x}_{\perp}\right)=Z \alpha \ln \vec{x}_{\perp}^{2}$ is given by the following closed expression:

$$
\begin{aligned}
\mathcal{T}\left(\vec{k}_{\perp}\right) & =\left(\frac{4}{\vec{k}_{\perp}^{2}}\right)^{1-i \alpha Z} \Gamma^{2}(1-i \alpha Z) \sin (\pi i \alpha Z) \\
& =\pi \frac{\Gamma(1-i \alpha Z)}{\Gamma(i \alpha Z)}\left(\frac{4}{\vec{k}_{\perp}^{2}}\right)^{1-i \alpha Z} .
\end{aligned}
$$

The first terms of the Taylor expansion read

$$
\begin{aligned}
\mathcal{T}\left(\vec{k}_{\perp}\right) \approx & +4 \pi i \alpha Z \frac{1}{\vec{k}_{\perp}^{2}}+4 \pi(i \alpha Z)^{2} \frac{\ln \left(\vec{k}_{\perp}^{2} / 4\right)+C}{\vec{k}_{\perp}^{2}} \\
& +2 \pi(i \alpha Z)^{3} \frac{\ln ^{2}\left(\vec{k}_{\perp}^{2} / 4\right)+4 C \ln \left(\vec{k}_{\perp}^{2} / 4\right)+4 C^{2}}{\vec{k}_{\perp}^{2}}+\cdots
\end{aligned}
$$

The second term would then correspond to the desired Fourier transform, the third term correspondingly to the Fourier transform of the square of the potential (A8), which has to be compared with the result of Torgerson [2].

This is, however, not justified, since the linearity of the Fourier transform is only guaranteed for finite sums and causes problems when applied to infinite series like the Taylor expansion of the exponential function. To get the correct result for the exact two-dimensional Euclidean photon propagator, the limit $i Z \alpha \rightarrow 0$ in 


$$
\begin{aligned}
& \int d^{2} x_{\perp} e^{-i \vec{k}_{\perp} \cdot \vec{x}_{\perp} \ln \vec{x}_{\perp}^{2}} \\
& =\lim _{i Z \alpha \rightarrow 0} \frac{d}{d(i Z \alpha)}\left[\pi \frac{\Gamma(1-i \alpha Z)}{\Gamma(i \alpha Z)}\left(\frac{4}{\vec{k}_{\perp}^{2}}\right)^{1-i \alpha Z}\right]
\end{aligned}
$$

has to be taken after having integrated the result with a test function [20]. Performing the limit without this precaution gives the wrong result (D3).

Another form of the correct Fourier transform was derived in [21,22]. We obtain the equivalent form from the gauged potential $A_{0}^{\prime}$ (A7). Since

$$
\ln x_{\perp}^{2}=\lim _{\gamma \rightarrow \infty} \int_{-\epsilon}^{\epsilon} d t\left(\frac{-\gamma}{\sqrt{\gamma^{2} t^{2}+\vec{x}_{\perp}^{2}}}+\frac{\gamma}{\sqrt{\gamma^{2} t^{2}+1}}\right)
$$

( $\epsilon$ is arbitrary but finite) and

$$
\begin{gathered}
\int d t d^{2} x_{\perp} e^{i \omega t-i \vec{k}_{\perp} \cdot \vec{x}_{\perp}}\left(\frac{\gamma}{\sqrt{\gamma^{2} t^{2}+\vec{x}_{\perp}^{2}}}-\frac{\gamma}{\sqrt{\gamma^{2} t^{2}+1}}\right) \\
=4 \pi\left[\frac{1}{\left(\frac{\omega}{\gamma}\right)^{2}+\vec{k}_{\perp}^{2}}-2 \pi \delta^{2}\left(\vec{k}_{\perp}\right) K_{0}\left(\frac{\omega}{\gamma}\right)\right],
\end{gathered}
$$

we find, by direct substitution,

$$
\begin{aligned}
\int d^{2} x_{\perp} e^{-i \vec{k}_{\perp} \cdot \vec{x}_{\perp} \ln \vec{x}_{\perp}^{2}}= & \lim _{\lambda \rightarrow 0} 4 \pi\left[\frac{-1}{\vec{k}_{\perp}^{2}+\lambda^{2}}\right. \\
& \left.+\pi \delta^{2}\left(\vec{k}_{\perp}\right) \ln \left(\frac{\mu^{2}}{\lambda^{2}}\right)\right]
\end{aligned}
$$

with $\lambda=\omega / \gamma, \mu=2 / e^{C}$. The limit has to be treated in the same way as in Eq. (D8).
[1] H.D.I. Abarbanel and C. Itzykson, Phys. Rev. Lett. 23, 53 (1969).

[2] R. Torgerson, Phys. Rev. 143, 1194 (1966).

[3] R. Jackiw, D. Kabat, and M. Ortiz, Phys. Lett. B 277, 148 (1992).

[4] G. 't Hooft, Phys. Lett. B 198, 61 (1987).

[5] S.J. Chang and S.K. Ma, Phys. Rev. 188, 2385 (1969); Phys. Rev. Lett. 22, 1336 (1969).

[6] R.L. Sugar and R. Blankenbecler, Phys. Rev. 183, 1387 (1969).

[7] M. Levy and J. Sucher, Phys. Rev. 186, 1656 (1969).

[8] S.J. Chang and P.M. Fishbane, Phys. Rev. D 2, 1104 (1970).

[9] B. Segev and J.C. Wells, Phys. Rev. A 57, 1849 (1998).

[10] C. Bottcher and M.R. Strayer, Phys. Rev. D 39, 1330 (1989).

[11] D.C. Ionescu and J. Eichler, Phys. Rev. A 48, 1176 (1993).

[12] R.M. Miura, J. Math. Phys. 9, 1202 (1968).

[13] P.C. Aichelburg and R.U. Sexl, Gen. Relativ. Gravit. 2, 303 (1971).

[14] H.M. Fried, Functional Methods and Models in Quantum Field Theory (MIT Press, Cambridge, MA, 1972).

[15] W. Magnus, Commun. Pure Appl. Math. 7, 649 (1954).

[16] E. Meggiolaro, Phys. Rev. D 53, 3835 (1996).

[17] L.D. Landau and E.M. Lifschitz, Lehrbuch der Theoretischen Physik (Akademie, Berlin, 1991), Vol. III.

[18] C. Itzykson and J.B. Zuber, Quantum Field Theory (McGrawHill, New York, 1985).

[19] A.J. Baltz, Phys. Rev. Lett. 78, 1231 (1997).

[20] G. Grignani and M. Mintchev, Phys. Rev. D 38, 3163 (1988).

[21] R. Ferrari, Nuovo Cimento A 19, 204 (1974).

[22] H. Balasin, W. Kummer, O. Piguet, and M. Schweda, Phys. Lett. B 287, 138 (1992).

[23] B. Segev and J.C. Wells, e-print physics/9805013.
[24] C.R. Vane et al., Phys. Rev. A 50, 2313 (1994); 56, 3682 (1997).

[25] H. Cheng and T.T. Wu, Expanding Protons: Scattering at High Energies (MIT Press, Cambridge, MA, 1987).

[26] A.J. Baltz and L. McLerran, Phys. Rev. C 58, 1679 (1998).

[27] K. Hencken, D. Trautmann, and G. Baur, Phys. Rev. A 51, 1874 (1995).

[28] M.C. Güçlü, J.C. Wells, A.S. Umar, M.R. Strayer, and D.J. Ernst, Phys. Rev. A 51, 1836 (1995).

[29] L.D. Landau and E.M. Lifschitz, Lehrbuch der Theoretischen Physik (Ref. [17]), Vol. IV.

[30] S.J. Brodsky, T. Kinoshita, and H. Terazawa, Phys. Rev. D 4, 1532 (1971).

[31] M. Vidovic, M. Greiner, C. Best, and G. Soff, Phys. Rev. C 47, 2308 (1993).

[32] S. Marculescu and L. Banyai, Rev. Roum. Phys. 24, 843 (1979).

[33] J.D. Jackson, Classical Electrodynamics (Wiley, New York, 1975).

[34] Handbook of Mathematical Functions, Natl. Bur. Stand. Appl. Math. Ser. No. 55, edited by M. Abramowitz and I.A. Stegun (U.S. GPO, Washington, D.C., 1964).

[35] M. Drees, J. Ellis, and D. Zeppenfeld, Phys. Lett. B 223, 454 (1989).

[36] C. Best, W. Greiner, and G. Soff, Phys. Rev. A 46, 261 (1992).

[37] M.J. Rhoades-Brown and J. Weneser, Phys. Rev. A 44, 330 (1991).

[38] M.J. Lighthill, Introduction to Fourier Analysis and Generalized Functions (Cambridge Univ. Press, London, 1959).

[39] A.J. Baltz, Phys. Rev. A 52, 4970 (1995).

[40] N. Toshima and J. Eichler, Phys. Rev. A 42, 3896 (1990). 